\title{
PHOTOMETRY OF VARIABLE STARS FROM DOME A, ANTARCTICA
}

\author{
Lingzhi Wang ${ }^{1,2}$, Lucas M. Macri ${ }^{2}$, Kevin Krisciunas $^{2}$, Lifan Wang ${ }^{2,3}$, Michael C. B. Ashley $^{4}$, \\ XiangQun Cui ${ }^{5}$, Long-Long Feng ${ }^{3}$, Xuefei Gong ${ }^{5}$, Jon S. Lawrence ${ }^{4,6}$, Qiang Liu ${ }^{7}$, \\ Daniel Luong-Van ${ }^{4}$, Carl R. Pennypacker ${ }^{8}$, Zhaohui Shang ${ }^{9}$, John W. V. Storey ${ }^{4}$, Huigen Yang ${ }^{10}$,

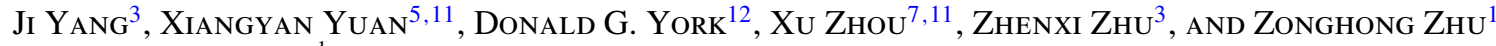 \\ ${ }^{1}$ Department of Astronomy, Beijing Normal University, Beijing 100875, China \\ ${ }^{2}$ Mitchell Institute for Fundamental Physics \& Astronomy, Department of Physics \& Astronomy, Texas A\&M University, College Station, TX 77843, USA \\ ${ }^{3}$ Purple Mountain Observatory, Chinese Academy of Sciences, Nanjing 210008, China \\ ${ }^{4}$ School of Physics, University of New South Wales, NSW 2052, Australia \\ ${ }^{5}$ Nanjing Institute of Astronomical Optics and Technology, Nanjing 210042, China \\ ${ }^{6}$ Australian Astronomical Observatory, NSW 1710, Australia \\ ${ }^{7}$ National Astronomical Observatory of China, Chinese Academy of Sciences, Beijing 100012, China \\ ${ }^{8}$ Center for Astrophysics, Lawrence Berkeley National Laboratory, Berkeley, CA, USA \\ ${ }^{9}$ Department of Physics, Tianjin Normal University, Tianjin 300074, China \\ ${ }^{10}$ Polar Research Institute of China, Pudong, Shanghai 200136, China \\ ${ }^{11}$ Chinese Center for Antarctic Astronomy, Nanjing 210008, China \\ 12 Department of Astronomy and Astrophysics and Enrico Fermi Institute, University of Chicago, Chicago, IL 60637, USA \\ Received 2011 July 6; accepted 2011 July 27; published 2011 October 3
}

\begin{abstract}
Dome A on the Antarctic plateau is likely one of the best observing sites on Earth thanks to the excellent atmospheric conditions present at the site during the long polar winter night. We present high-cadence time-series aperture photometry of 10,000 stars with $i<14.5$ mag located in a $23 \mathrm{deg}^{2}$ region centered on the south celestial pole. The photometry was obtained with one of the CSTAR telescopes during 128 days of the 2008 Antarctic winter. We used this photometric data set to derive site statistics for Dome A and to search for variable stars. Thanks to the nearly uninterrupted synoptic coverage, we found six times as many variables as previous surveys with similar magnitude limits. We detected 157 variable stars, of which 55\% were unclassified, 27\% were likely binaries, and $17 \%$ were likely pulsating stars. The latter category includes $\delta$ Scuti, $\gamma$ Doradus, and RR Lyrae variables. One variable may be a transiting exoplanet.
\end{abstract}

Key words: site testing - stars: variables: general

Online-only material: color figures, machine-readable and VO tables

\section{INTRODUCTION}

Long, continuous, unbroken time-series photometry is highly advantageous for a range of astrophysical problems, such as exoplanet searches, studies of periodic variable stars, and discoveries of previously unknown stellar behavior. While these data can be obtained via coordinated observations by a worldwide telescope network, such an approach is fraught with calibration issues and variable weather across the sites, and is highly labor intensive. Ideally, the data would be acquired with a single, fully autonomous robotic telescope. Space is obviously an ideal place to acquire such data due to the high photometric quality and long observing sequences that can be achieved, as demonstrated by the CoRoT (Baglin et al. 2006; Boisnard \& Auvergne 2006) and Kepler missions (Benkő et al. 2010; Borucki et al. 2010). Antarctica offers the only location on the surface of the Earth where similar observations can be obtained.

The Antarctic plateau offers an unparalleled opportunity to make these types of observations with a single telescope. The combination of high altitude, low temperature, low absolute humidity, low wind, and extremely stable atmosphere opens new windows in the infrared and terahertz regions and offers improved conditions at optical and other wavelengths. These advantages offer astronomers gains in sensitivity and measurement precision that can exceed two orders of magnitude over even the best temperate sites (Storey 2005, 2007, 2009; Burton 2010). Additionally, the high duty cycle and long duration of observations from Antarctic sites such as Dome $\mathrm{A}$ and Dome $\mathrm{C}$ allow for efficient asteroseismic observations. Observations with one site on the Antarctic plateau offer a performance similar to or better than a six-site network at other latitudes (Mosser \& Aristidi 2007).

Furthermore, sites on the Antarctic plateau suffer from less high-altitude turbulence than temperate sites and thus enjoy lower scintillation noise leading to superior photometric precision (Kenyon et al. 2006). The elevation of a source observed from Antarctica changes little during the course of $24 \mathrm{hr}$, again improving photometric precision and providing the potential for long, continuous time-series observations.

Other advantages include dark skies and lower precipitable water vapor (PWV) which lead to better atmospheric transmission. Some disadvantages that must be considered include aurorae, a reduced amount of the celestial sphere that is available for observations, and prolonged twilight. One site on Antarctica, Dome C, has a similar amount of cloud-free astronomically dark time and has lower atmospheric scattering than low-latitude sites, reducing the sky brightness and extinction (Kenyon \& Storey 2006).

A very promising site on the Antarctic plateau is Dome A, at an elevation of $4093 \mathrm{~m}$ (840 m higher than Dome C), where the Chinese Kunlun station is under construction. Dome A is possibly the best astronomical site on Earth based on data, models, and meteorological parameters such as cloud cover, boundary layer characteristics, aurorae, airglow, free atmosphere, and PWV (Saunders et al. 2009, 2010). Swain \& Gallée (2006) have modeled the turbulent surface layer across the entire Antarctic ice sheet and found thickness differences from site to site, with one of the thinnest regions being at Dome A. This was confirmed 
Table 1

Log of Observations

\begin{tabular}{lrr}
\hline \hline $\begin{array}{l}\text { Month } \\
2008\end{array}$ & No. of Images & $\begin{array}{r}\text { Total Exp. } \\
\text { Time (hr) }\end{array}$ \\
\hline March & 14345 & 64.4 \\
April & 51390 & 316.4 \\
May & 71665 & 398.1 \\
June & 110358 & 613.1 \\
July & 40082 & 222.7 \\
Total & 287840 & 1614.7 \\
\hline
\end{tabular}

by Snodar measurements in 2009. The median thickness of the boundary layer was $14 \mathrm{~m}$ (Bonner et al. 2010).

Long-term time-series photometric data have been acquired at the South Pole and at Dome C. The $5 \mathrm{~cm}$ South Pole Optical Telescope was used to obtain continuous observations over 78 hr of the Wolf-Rayet star $\gamma^{2}$ Velorum (Taylor 1990). Strassmeier et al. (2008) used the $25 \mathrm{~cm}$ sIRAIT telescope to obtain continuous ( $98 \%$ duty cycle over 10 days) photometry of two bright variable stars. The resulting light curves had rms scatter of $3 \mathrm{mmag}$ in the $V$ band and $4 \mathrm{mmag}$ in the $R$ band over a period of $2.4 \mathrm{hr}$, values that are 3-4 times better than previously obtained with an equivalent telescope in southern Arizona. They attributed the improved photometric precision to the exceptionally low scintillation noise in the Antarctic plateau. The ASTEP project (Crouzet et al. 2010) used a fixed $10 \mathrm{~cm}$ refractor to monitor a $15 \mathrm{deg}^{2}$ field centered on the south celestial pole during four months of operation (2008 June-September). They found that sky conditions suitable for observations were present for $\sim 85 \%$ of the time and calculated an observing efficiency for the detection of short-period exoplanets around bright stars that is a factor of 1.5 times higher than that of temperate sites.

An observatory that can operate year round without interruptions is required to best capitalize upon the advantages provided by the Antarctic plateau. We have built such an observatory at Dome A, called PLATO (PLATeau Observatory; Ashley et al. 2010; Luong-van et al. 2010; Yang et al. 2009; Lawrence et al. 2009; Lawrence et al. 2008; Hengst et al. 2008; Lawrence et al. 2006), and a quad-telescope called CSTAR (the Chinese Small Telescope ARray; Yuan et al. 2008; Zhou et al. 2010b). Based on a large amount of high-quality photometric data obtained during the 2008 Antarctic winter, Zou et al. (2010) undertook a variety of sky brightness, transparency, and photometric monitoring observations, while Zhou et al. (2010a) published a catalog of $\sim 10,000$ stars in a field centered on the south celestial pole.

This paper presents an independent analysis of the data acquired by CSTAR during the 2008 Antarctic winter season. Section 2 briefly describes the instrument, observations, and data reduction; Section 3 presents details of the photometric procedure and the astrometric and photometric calibrations; Section 4 describes the steps followed to obtain high-precision time-series photometry of the brightest 10,000 stars; Section 5 presents a catalog of variable stars and rough statistics of variable star types; Section 6 contains our conclusions.

\section{OBSERVATIONS AND DATA REDUCTION}

\subsection{Observations}

CSTAR (Yuan et al. 2008; Zhou et al. 2010b), a part of the PLATO observatory, ${ }^{13}$ is the first photometric instrument

\footnotetext{
13 http://mcba11.phys.unsw.edu.au/ plato/cstar.html
}

Table 2

Exposure Times

\begin{tabular}{lccr}
\hline \hline $\begin{array}{l}\text { Start } \\
\text { Date }\end{array}$ & $\begin{array}{c}\text { End } \\
\text { Date }\end{array}$ & $\begin{array}{c}\text { Exp. } \\
(\mathrm{s})\end{array}$ & $\begin{array}{r}\text { No. of } \\
\text { Images }\end{array}$ \\
\hline 2008 Mar 20 & 2008 Mar 23 & 5 & 7944 \\
2008 Mar 23 & 2008 Apr 11 & 30 & 17514 \\
2008 Apr 11 & 2008 Jul 27 & 20 & 262382 \\
\hline
\end{tabular}

deployed at Dome A. It was built by astronomers Xu Zhou and Zhenxi Zhu and is composed of four Schmidt-Cassegrain wide field telescopes. Each CSTAR telescope has a field of view 4.5 in diameter with a pupil entrance aperture of $145 \mathrm{~mm}$. Each focal plane contains an ANDOR DV435 $1 \mathrm{~K} \times 1 \mathrm{~K}$ frametransfer CCD with a pixel size of $13 \mu \mathrm{m}$, which translates to a plate scale of $15^{\prime \prime}$ pixel $^{-1}$. Three of the telescopes have $g, r$, and $i$ filters similar to those used by Sloan Digital Sky Survey (Fukugita et al. 1996). Table 1 of Zhou et al. (2010b) lists the effective wavelength $(470 \mathrm{~nm}, 630 \mathrm{~nm}, 780 \mathrm{~nm})$ and full width at half-maximum $(140 \mathrm{~nm}, 140 \mathrm{~nm}, 160 \mathrm{~nm})$ of the three filters $(g, r, i)$. No filter is used in the fourth telescope. The field of view of the telescopes is very nearly centered on the south celestial pole. Since the telescopes are fixed, stars move in circles about the center of the CCD but only traverse a tiny fraction of a pixel during a single exposure. More details on CSTAR can be found in Zhou et al. (2010a, 2010b).

The CSTAR telescopes were installed at Dome A in 2008 January. During the following Antarctic winter season, there were technical problems with three of the four telescopes- those that use the Sloan $g$, Sloan $r$, and "open" filters-which prevented them from obtaining any useful data. Fortunately, the fourth telescope (equipped with the Sloan $i$ filter) performed without any significant issues. Observations were conducted from 2008 March 20 through 2008 July 27; during this interval, more than 287,800 frames were acquired with a total integration time of $1615 \mathrm{hr}$. The total amount of raw data collected during the observing season was about 350 GB. Table 1 lists the number of images acquired and total exposure time per month, while Table 2 details the different exposure times used during the observing season.

Two groups have carried out independent analyses of the data; one at the National Astronomical Observatories of the Chinese Academy of Sciences (Zhou et al. 2010a) and another at Texas A\&M University and Beijing Normal University (present work). A comparison of the photometric precision of the two reductions is given in Section 4.2.

\subsection{Data Reduction}

The preliminary reduction for the raw science images involved bias subtraction, flat fielding, and fringe correction. We used a bias frame obtained during instrument testing in China. We created a sky flat by median combining 160 images with high sky levels ( $\geqslant 15,000$ ADU) obtained on 2008 March 17.

The bias-subtracted and flat-fielded $i$-band images contained a residual fringe pattern with peak-to-peak variations of $\sim 1.4 \%$ of the sky value. The fringes are due to variations in the thickness of the CCD and strong emission lines in this region of the visible spectrum. An important aspect of these features is that they introduce an additive contribution to the signal and they should be subtracted to perform photometrically consistent measurements. We created a fringe correction image by carrying out a two-step process described below. 
We selected 3450 bias-subtracted and flat-fielded images obtained during 2008 March which exhibited a strong fringe pattern. We first masked all saturated pixels (values above 25,000 ADU) and their nearest neighbors. We then carried out point-spread function (PSF) photometry using an automated pipeline based on DAOPHOT and ALLSTAR (Stetson 1987). We set the detection threshold to $5 \sigma$ above sky. We masked the pixels associated with every star detected in each frame, using different radii for different ranges of instrumental magnitude (15 pixels for $m<15 \mathrm{mag}, 10$ pixels for $15<m<17 \mathrm{mag}$, and 7 pixels for $m>17 \mathrm{mag}$ ). All remaining pixels on the masked images represented contributions from the sky background or stars below our detection threshold (see below). We grouped the images into 23 sets of 150 frames each, placing consecutive images in different sets to minimize the overlap of masked regions. Since the CSTAR telescopes do not track the rotation of the sky, interleaving consecutive images into different sets ensures that the pixels masked due to the presence of stars will fall at different positions on the detector. We normalized every image by its median sky value and median combined each set. We then median combined the 23 intermediate images into an initial fringe correction image.

The initial fringe correction image could be affected by faint stars below the $5 \sigma$ detection threshold used in the above procedure. In order to remove such objects, we applied a preliminary fringe correction to the input frames and lowered the detection threshold to $2.5 \sigma$ above sky. We merged the masks obtained in each of the two steps and repeated the combination process described above to obtain a final fringe correction image.

The fringe correction image was subtracted from each science-quality frame in an iterative manner, scaling its amplitude until the residuals in two corner areas of the frame were statistically indistinguishable.

\section{PHOTOMETRY}

\subsection{Frame Selection and Photometry}

We performed photometry on the debiased, flattened, fringecorrected images using a pipeline based on DAOPHOT and ALLSTAR. We used the FIND and SKY routines in the IDL version of DAOPHOT to reject images taken under very high sky background or cloudy conditions. We selected frames with a sky level below 6000 ADU and more than 1500 stars in the frame; these criteria were met by $93 \%$ of the images (about 270,000 frames). Figure 1 shows the distribution of the selected and rejected frames as a function of date and Sun elevation angle. Forty-nine percent of the data obtained in March was rejected due to the high sky background caused by the relatively large Sun elevation angle. Only $4 \%, 5 \%, 1 \%$, and $11 \%$ of the data acquired in April, May, June, and July, respectively, were rejected. Based on Figure 1, a suitable initial cutoff for useful $i$-band data at Dome A would be a solar elevation angle below $-10^{\circ}$. Ninety-six percent of all frames obtained at or below that elevation angle passed our selection criteria.

Frame-by-frame registration was used to combine 3000 of the best images obtained during a $24 \mathrm{hr}$ period of exceptionally good conditions (2008 June 29) to form a master reference image. We selected images with sky values below 500 ADU and with more than 10,000 stars above the detection threshold. We combined the images using MONTAGE (developed by P. Stetson) into a master image that was resampled (via bicubic interpolation) at four times the initial pixel scale. We restricted the master

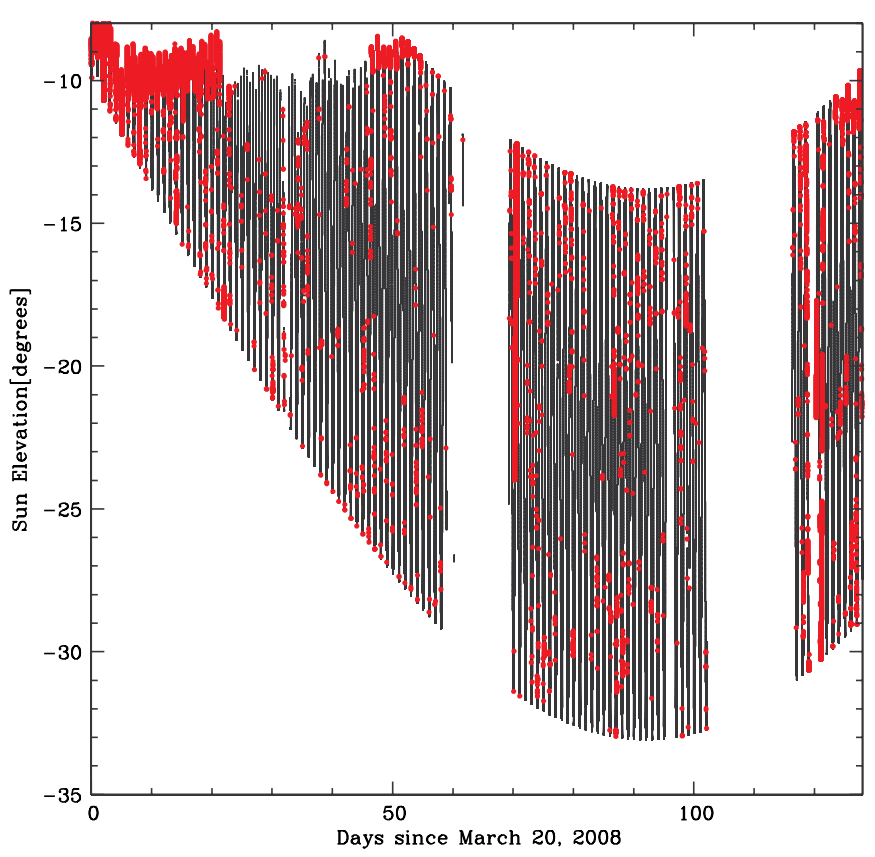

Figure 1. Sun elevation angle for each science-quality frame obtained with CSTAR during the Antarctic winter of 2008. Black points indicate frames suitable for analysis, while red points denote frames that failed to meet our selection criteria.

(A color version of this figure is available in the online journal.)

frame to a circular field of view that was observed continuously, spanning $-90^{\circ}<\delta<-87^{\circ} 17^{\prime}$. We masked saturated stars and their surroundings using a circular mask of varying radius. We identified all stars in the masked master image using DAOFIND and a detection threshold of $5 \sigma$. The master image is shown in Figure 2.

Next, we carried out aperture and PSF photometry using the stand-alone versions of DAOPHOT and ALLSTAR. The aperture photometry radius was set to 2.5 pixels (37'.5), with the sky annulus extending from 4 to 7 pixels $\left(60^{\prime \prime}-105^{\prime \prime}\right)$. Figure 3 shows the radial profile and the location of these radii for a random star.

The smallest uncertainties reported by DAOPHOT for aperture photometry were of the order of 2 mmag. We modeled the PSF using a Moffat function with $\beta=1.5$, which gave the smallest residuals. Due to the severe undersampling of the camera $\left(15^{\prime \prime}\right.$ pixel $\left.^{-1}\right)$, the smallest uncertainties reported by ALLSTAR for PSF photometry were significantly larger $(\sim 0.05 \mathrm{mag})$ than those reported for aperture photometry. Figure 4 shows a comparison between the reported DAOPHOT aperture and PSF photometric uncertainties. We conclude that PSF photometry is not suitable for the analysis of CSTAR data, and we only use aperture photometry for the remainder of this paper.

We carried out aperture photometry on selected scientific images to determine for each one the number of stars, the sky level, and the photometric zero point (proxy for extinction due to clouds) relative to the master frame. The latter was calculated from the magnitude offset of the brightest 1000 stars in each image across the whole field of view with respect to the corresponding magnitudes in an individual image chosen as our internal photometric reference. Outlier rejections of $3 \sigma$ were applied iteratively to guarantee that the zero points were based on non-variable stars. Figure 5 shows the time series of number of stars, sky level, and photometric zero point (proxy for differential extinction due to clouds), while Figure 6 shows the 


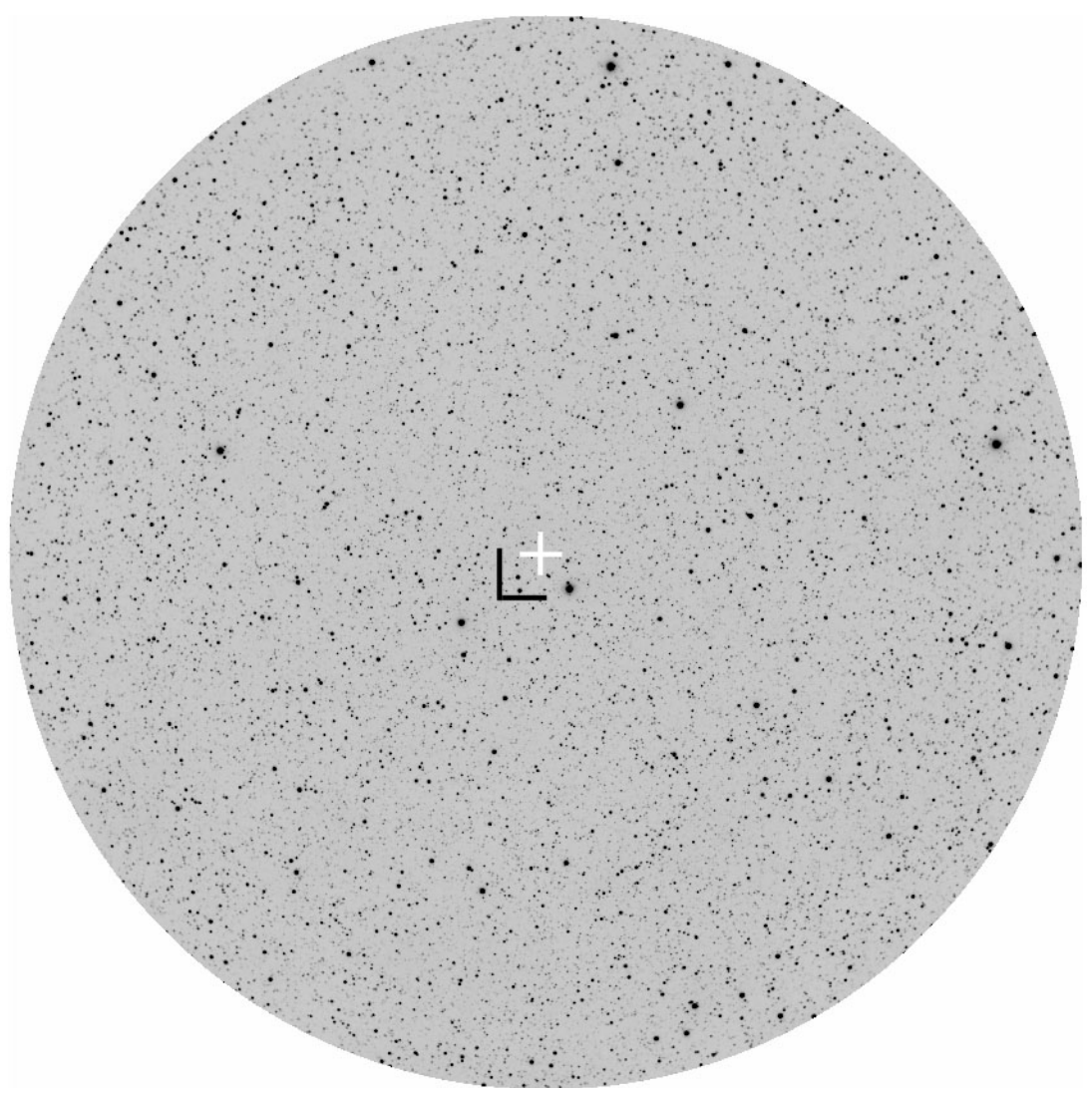

Figure 2. Master frame of the CSTAR field of view. The white cross denotes the position of the south celestial pole. The edge of the field corresponds to $\delta=-87^{\circ} 17^{\prime}$. The black lines indicate the bottom-left edge of image shown in Figure 3, while the top-right edge is close to the SCP.

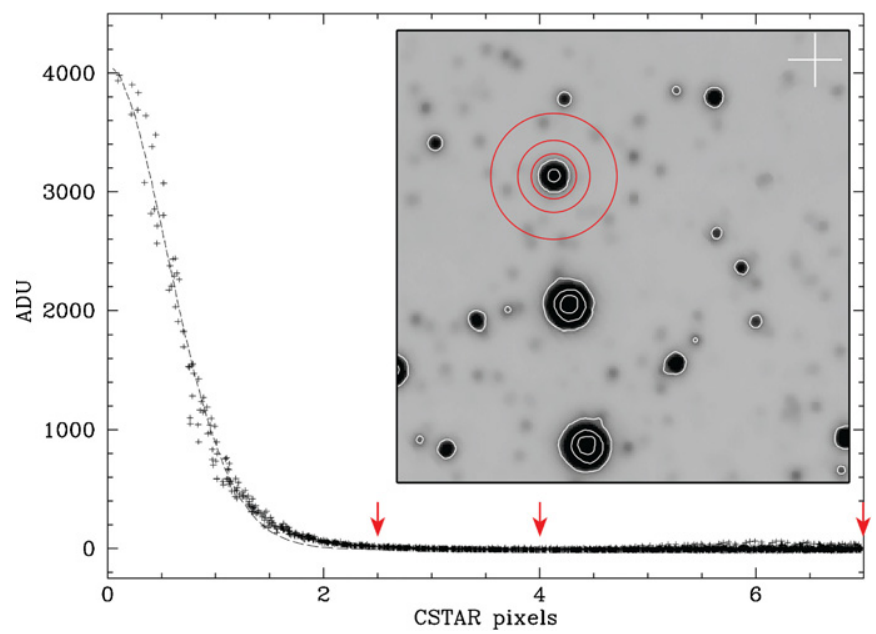

Figure 3. Inset: zoom into a small region of the master image of the CSTAR field ( $12^{\prime}$ on a side) near the south celestial pole (indicated by the white cross as in Figure 2). The white contours indicate intensity levels logarithmically spaced between 20 and $10^{4} \mathrm{ADU}$. The red circles indicate the extent of the aperture radius and the sky annulus, centered on a random star. Outer plot: radial profile for the same star, indicating the aperture radius as well as the inner and outer radii of the sky annulus. The pixel scale is $15^{\prime \prime}$ pixel $^{-1}$.

(A color version of this figure is available in the online journal.)

frequencies of occurrence and cumulative density distributions of the same quantities.

There is a small zero-point offset of $\sim 0.03$ mag between our reference image and the one used by Zou et al. (2010). Hence, the peak value of the distribution in the top right panel in Figure 6 is shifted to the left by that amount relative to Figure

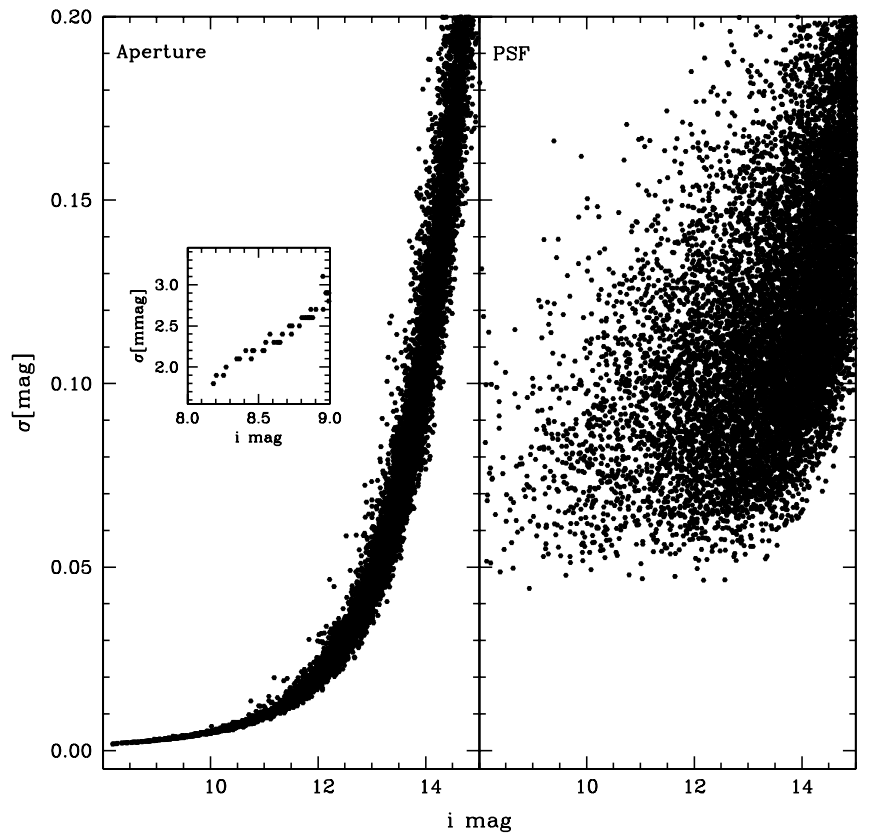

Figure 4. Comparison of DAOPHOT/ALLSTAR uncertainties for aperture (left) and PSF (right) magnitudes for a typical frame. The inset zooms in the aperture photometry for $8<m<9$.

5 of Zou et al. (2010). Based on these values, we can conclude that extinction due to clouds in the $i$ band at Dome A is less than 0.4 mag during $80 \%$ of the time and less than $0.1 \mathrm{mag}$ for $50 \%$ of the time. Our results are consistent with those presented in Table 1 of Zou et al. (2010). 

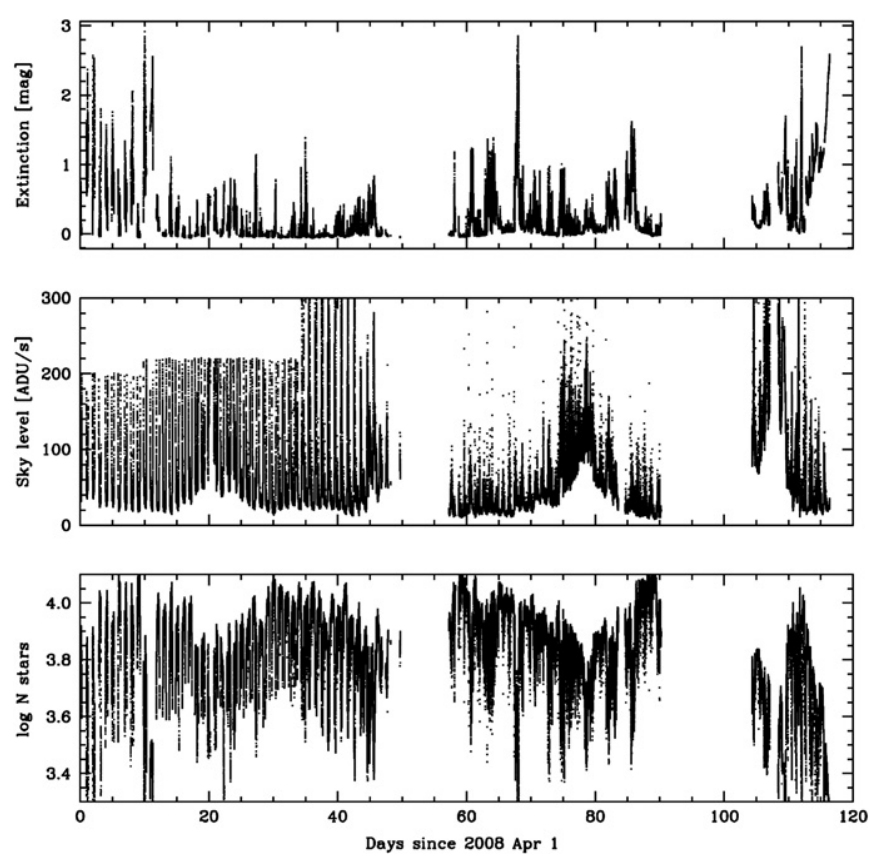

Figure 5. Time-series plots of differential extinction (top panel), sky brightness level (middle panel), and number of stars on a single image (bottom panel).

Fifty percent of the science-quality images have at least 7500 stars above the detection threshold and a sky level below $36 \mathrm{ADU} \mathrm{s}^{-1}$. The latter is equivalent to a median sky background of $19.6 \mathrm{mag}$ per $\operatorname{arcsec}^{2}$, consistent with the value of $19.8 \mathrm{mag}$ per $\operatorname{arcsec}^{2}$ derived by Zou et al. (2010). That paper also compared the median sky background from La Palma, Paranal, Cerro Tololo, and Calar Alto and concluded that under moonless clear conditions Dome A has darker sky backgrounds than the above astronomical sites.

\subsection{Astrometric and Photometric Calibration}

We determined the astrometric solution of our master reference image based on an independent astrometric calibration of a single frame (15CC0007) obtained during the same observing season by M. Ashley (2008, private communication). We found 5300 stars in common between this frame and our master reference image using DAOMATCH and DAOMASTER (developed by P. Stetson). We extracted the celestial coordinates of these stars from the calibrated frame using the "xy2sky" routine in WCSTools $^{14}$ and determined the astrometric solution of our master reference image using the routines "ccmap" and "ccsetwcs" in IRAF. ${ }^{15}$ ccmap was used to compute plate solutions using matched pixel and celestial coordinate lists and ccsetwcs was used to create an image world coordinate system from a plate solution. We used the TNX projection for the WCS of our master reference image.

We determined a mean astrometry uncertainty of $\sim 5^{\prime \prime}$ $(\sim 1 / 3$ pixels) by comparing the coordinates we derived and those tabulated in the Guide Star Catalog (version 2.3; Lasker et al. 2008) for selected stars across the entire field of view of our master frame. Figure 7 shows the distribution of positional differences for these stars.

\footnotetext{
14 http://tdc-www.harvard.edu/wcstools

15 IRAF is distributed by the National Optical Astronomy Observatory, which is operated by the Association of Universities for Research in Astronomy, Inc., under cooperative agreement with the National Science Foundation.
}
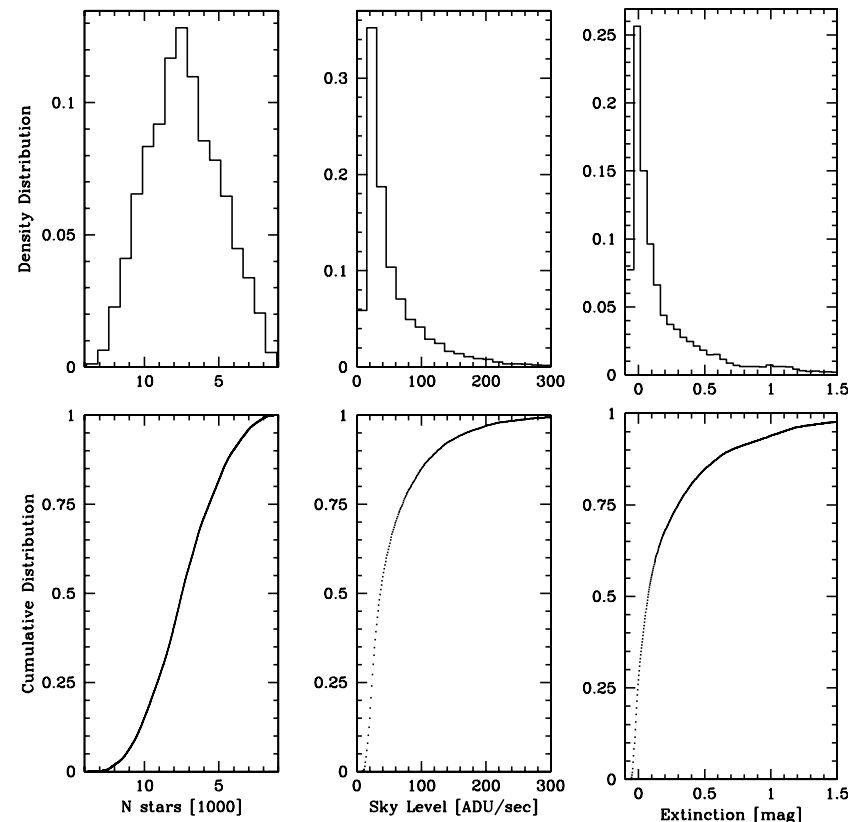

Figure 6. Frequency of occurrence and cumulative fraction of the number of stars per image (left column), sky brightness level (middle column), and differential extinction (right column).

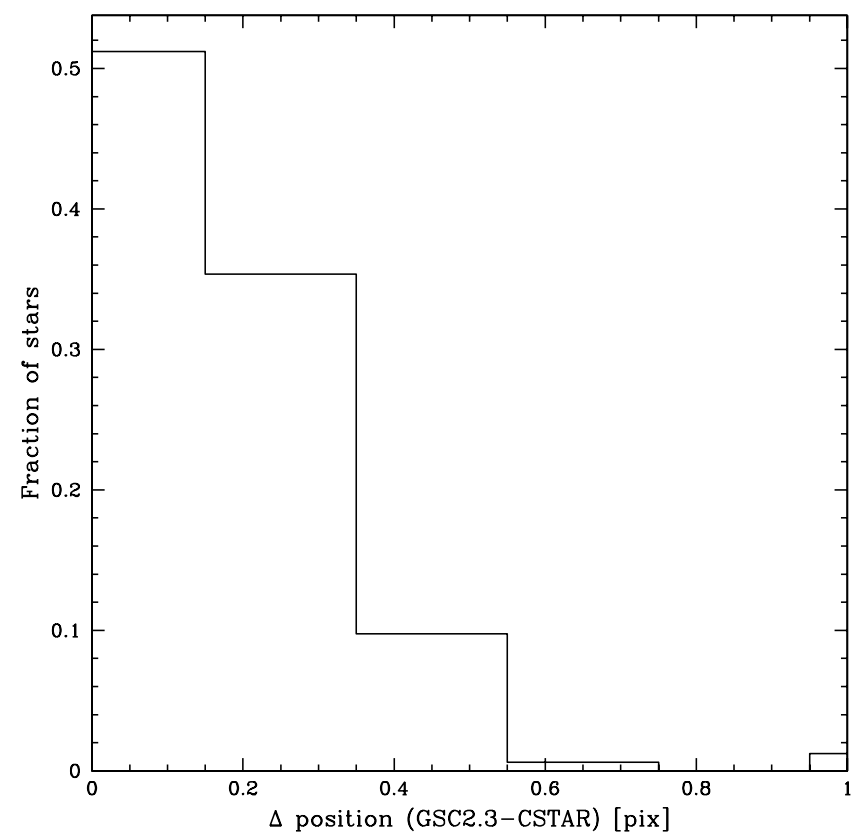

Figure 7. Distribution of astrometric differences between CSTAR and the Guide Star Catalog v2.3. The plate scale of CSTAR is $15^{\prime \prime}$ pixel $^{-1}$.

We determined the photometric calibration of our master reference image using the catalog of calibrated griz magnitudes of Tycho stars (Ofek 2008). We used Vizier ${ }^{16}$ to select stars with $9<i<11.5 \mathrm{mag}$ and $\delta<-87^{\circ}$ and matched them to objects in our master image using a positional tolerance of 2.5 pixels $\left(=37^{\prime \prime} .5\right)$. We applied an iterative outlier rejection and determined a photometric zero point of $7.46 \pm$ 0.08 mag based on $N=119$ stars, shown in the bottom panel of Figure 8.

We checked the above calibration by matching stars in our master frame with bright unblended stars in the catalog of

\footnotetext{
16 http://vizier.u-strasbg.fr
} 


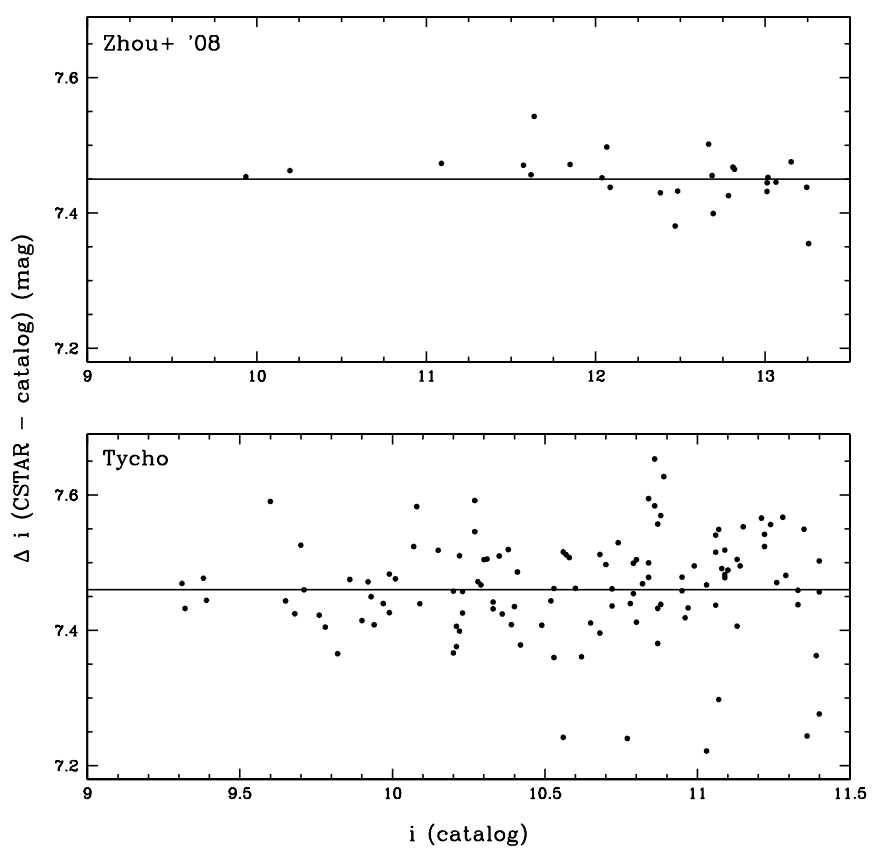

Figure 8. Photometric calibration of the CSTAR observations.

Zhou et al. (2010a), who computed aperture photometry using a 3 pixel radius. Those authors adopted a photometric calibration based on 48 stars in common with the USNO-B catalog, whose $i$-band magnitudes were determined by Monet et al. (2003). We determined a photometric zero point of $7.45 \pm 0.04 \mathrm{mag}$ (see top panel of Figure 8), which is statistically identical to the value determined above. We adopt the calibration based on the Tycho catalog, as it is based on a larger number of stars.

\section{TIME-SERIES PHOTOMETRY}

There are approximately 100,000 stars detected in our master reference image, reaching a depth of $i=20.4 \mathrm{mag}$. The brightest 10,000 of these are detected in most individual frames and correspond to the depth of a previous study of this area of the sky by the All-Sky Automated Survey (ASAS) project ( $V \sim 14.5$ mag; Pojmanski 2005). Hence, we selected these objects for time-series photometry with the aim of detecting variable stars and determining the most significant frequencies in their power spectra. Hereafter, we will refer to this subset as the "bright-star sample." We restricted our analysis to the images obtained under the best conditions, defined as a sky background below $50 \mathrm{ADU} \mathrm{s}^{-1}$ and extinction $\leqslant 0.5 \mathrm{mag}$. These conditions were met by 141,700 frames or about $53 \%$ of all the sciencequality data.

\subsection{Photometric Corrections}

We performed a series of corrections to the aperture photometry reported by DAOPHOT, which included exposure time normalization, zero-point correction, sigma rescaling, residual flat fielding, time calibration, masking of satellite trails and saturated regions, spike filtering, and magnitude calibration. In greater detail, these corrections entailed the following steps.

1. Exposure time normalization. The aperture photometry reported by DAOPHOT was corrected by the usual factor of $2.5 \log (t)$.
2. Zero-point correction. The zero-point difference between each image and the photometric reference image was determined following the procedure previously described in Section 3.

3. Sigma rescaling. We rescaled the magnitude uncertainties reported by DAOPHOT so that $\chi_{v}^{2}=1$ for non-variable stars, following the procedure developed by Kaluzny et al. (1998).

4. Residual flat fielding. We selected 200 bright non-variable stars and searched for correlations between their $(x, y)$ position and deviations from their mean magnitude on every science-quality frame. We averaged the results from neighboring pixels to increase signal-to-noise ratio $(\mathrm{S} / \mathrm{N})$, at the cost of decreasing the spatial resolution fourfold. The result is equivalent to a residual flat-field frame, which exhibited peak-to-peak variations of $1 \%$.

5. Time calibration. The clock in the data acquisition computer exhibited a small drift during the observing season. We corrected the Julian Dates of all science-quality frames following Section 4.3 of Zhou et al. (2010a).

6. Masking of satellite trails. Numerous satellite trails are present in the CSTAR images, easily identifiable as lines with fluxes $>10 \sigma$ above background. This polluted stars whose positions on the CCD overlapped the trails. We used a parallelogram mask with the trail line as the bisector and a width that encompassed all pixels with fluxes $>3 \sigma$ above background. This was equivalent to a width of 20 pixels or less, depending on the brightness of each trail. We rejected the individual photometric measurements of stars lying inside the masked regions of a given frame.

7. Masking of saturated regions. We masked pixels with values above 25,000 ADU and their associated bleed trails.

8. Spike filtering. We detected quasi-periodic, short-duration (typically 30 minutes) increases in the brightness of some stars (typically $0.4 \mathrm{mag}$ ) which were otherwise not variable. Some of these "spikes" were due to ghosts (multiple reflections of incoming photons) of bright stars which we identified by subtracting neighboring images. We initially identified spikes by searching for $3 \sigma$ deviations from the mean magnitude in $6 \mathrm{hr}$ blocks of data. In the case of periodic variable stars, the mean was computed over $1 / 8$ of the period or 0.1 day, whichever was smaller. We carried out the procedure twice, shifting the starting time of each bin by half of its width on the second pass. This algorithm might have eliminated some bona fide aperiodic flux variations (such as stellar flares), but we defer a thorough analysis of such events and other causes of these spikes to a future publication.

9. Magnitude calibration. We placed our corrected instrumental magnitudes on the standard system as described previously in Section 3.2.

\subsection{Photometric Precision}

Over $70 \%$ of the bright-star sample considered in this analysis has more than 20,000 photometric measurements. Thanks to this, the internal statistical uncertainty in the mean magnitudes of stars with $i \leqslant 13.5 \mathrm{mag}$ is less than $10^{-3} \mathrm{mag}$, as seen in Figure 9. As discussed previously in Section 3.2, the absolute uncertainty of the photometry is limited by the synthetic magnitude calibration using the Tycho catalog.

Figure 10 compares the scatter in our mean magnitudes with the results of Zhou et al. (2010a). We compared 27 stars in 


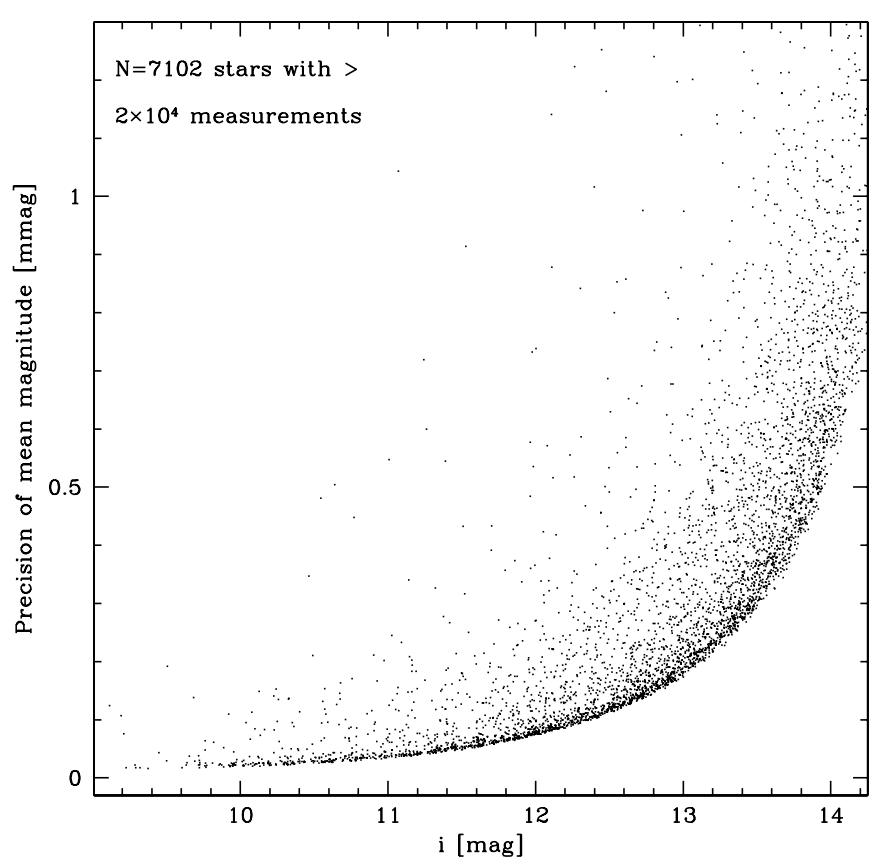

Figure 9. Photometric precision of mean $i$ magnitudes for more than 7000 stars that have at least 20,000 observations.

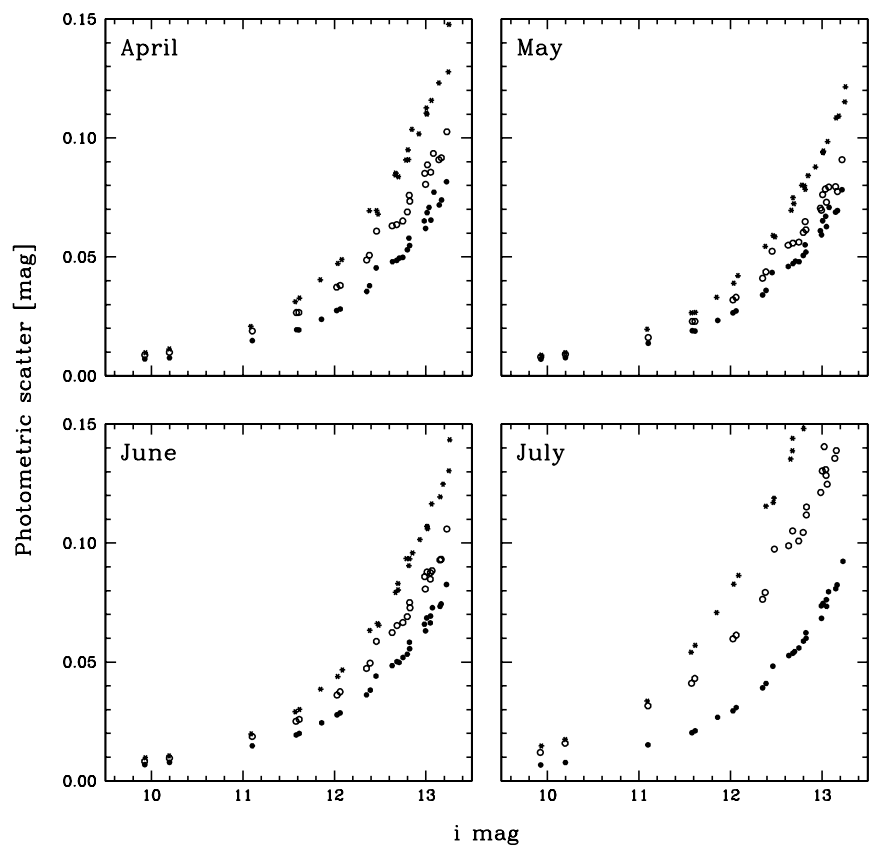

Figure 10. Comparison of photometric scatter for stars in common with Zhou et al. (2010a) that were used to determine the zero-point offset in the top panel of Figure 8. Separate comparisons are show for April, May, June, and July. Starred symbols: photometry from Zhou et al. (2010a); open symbols: our aperture photometry before applying the photometric corrections described in Section 4.1; filled symbols: our aperture photometry after applying the corrections.

common between our catalogs which were used to obtain the alternative zero point discussed in Section 3.2. We calculated the scatter in the mean magnitude of each star in both data sets using a $3 \sigma$ iterative outlier rejection, performing separate comparisons for each month of the observing season. Our mean magnitudes exhibit a slightly smaller scatter than Zhou et al. (2010a). Some possible reasons for the reduced scatter include the use of a smaller sky annulus located closer to each star and the rejection of measurements affected by satellite trails.

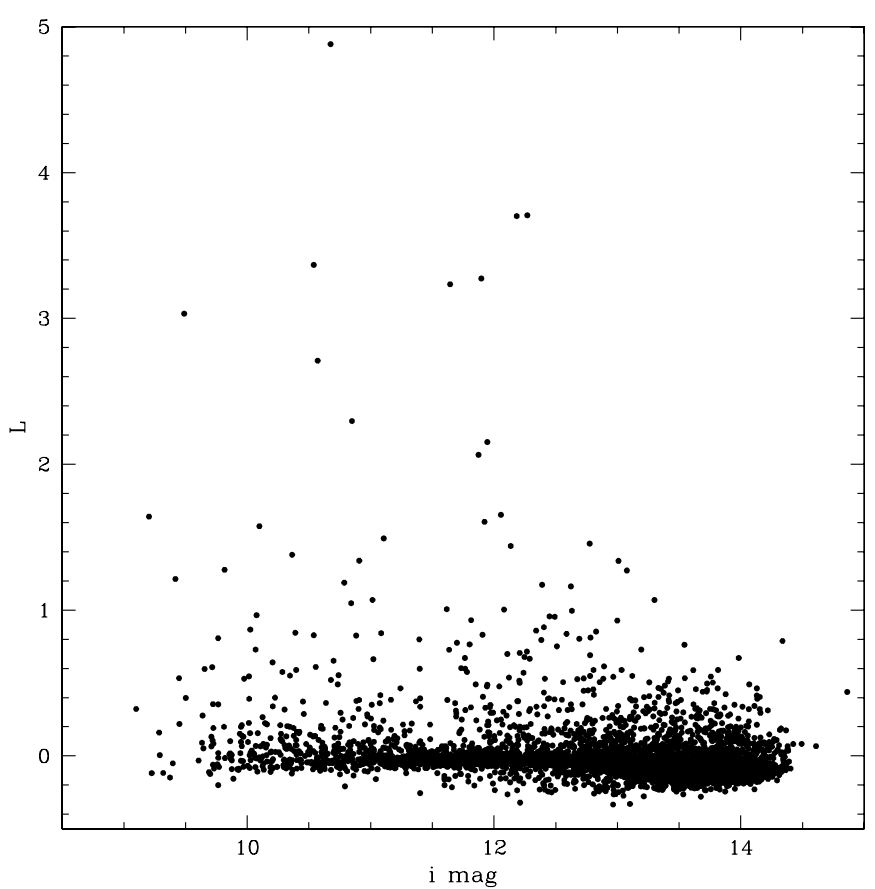

Figure 11. Variability statistic $L$ (Stetson 1996) for the 10,000 brightest stars in the CSTAR sample.

\section{VARIABLE STAR CATALOG AND STATISTICS}

\subsection{Search for Variability}

We applied several techniques to quantify the variability of the bright-star sample. First, we computed the Welch-Stetson variability index $L$ (Section 2 of Stetson 1996), which measures the time-dependent correlation of magnitude residuals of pairs of close observations, rescaling them according to the estimated standard error of each magnitude measurement. Figure 11 shows the distribution of $L$ values as a function of magnitude for this subsample.

We searched for periodic variability among the bright-star sample using three techniques. The first technique is the Lomb-Scargle method (Lomb 1976; Scargle 1982, hereafter LS), which applies the statistical properties of least-squares frequency analysis of unequally spaced data on a series of test periods. We searched for periods between 0.1 and 50 days and used a bin size of 0.01 days. Periods with $S / N \geqslant 10$ in the periodogram were considered to be significant. The second technique involves Fourier decomposition as implemented by the Period04 program (Lenz \& Breger 2005), which is described in detail in Section 5.2. We searched for frequencies between 0 and 50 cycles day ${ }^{-1}$ with a bin size set by the Nyquist sampling. Frequencies with $\mathrm{S} / \mathrm{N} \geqslant 4$ were considered significant.

Lastly, we searched for periodic transit events on prewhitened light curves using the box fitting algorithm (Kovács et al. 2002, hereafter BLS). This algorithm looks for signals characterized by a periodic alternation between two discrete levels with much less time spent at the low-level (occultation) phase. The duration of a transit was allowed to range between 0.01 and 0.1 of the primary period. The BLS transit period was sought over the same time span as the LS period using 10,000 trial periods and 200 phase bins. We prewhitened the selected light curves based on the most significant period $(1 / f)$ and its 10 higher-harmonics (frequencies of $2 f, 3 f, \ldots, 11 f$ ) and 9 subharmonics (frequencies of $f / 2, f / 3, \ldots, f / 10$ ). 
Table 3

Variable Stars

\begin{tabular}{|c|c|c|c|c|c|c|c|c|}
\hline \multicolumn{2}{|c|}{ ID } & \multirow[t]{2}{*}{ R.A. } & \multirow[t]{2}{*}{ Decl. } & \multirow{2}{*}{$\begin{array}{c}i \\
\text { (mag) }\end{array}$} & \multicolumn{2}{|c|}{ Period } & \multirow{2}{*}{$\begin{array}{c}T_{0}^{\mathrm{c}} \\
\text { (days) }\end{array}$} & \multirow[t]{2}{*}{ Type $^{\mathrm{d}}$} \\
\hline CSTAR & (GSC) & & & & (days) & $\left(\mathrm{Src}^{\mathrm{b}}\right)$ & & \\
\hline 000572 & S3YM000353 & $11: 11: 19.60$ & $-87: 18: 00.9$ & 13.88 & 0.154415 & LS & 55.3409 & DSCT \\
\hline 001707 & S74D000321 & $12: 32: 42.91$ & $-87: 26: 22.9$ & 11.07 & 0.338528 & LS & 56.5058 & $\mathrm{EC},[\mathrm{A}]$ \\
\hline 003125 & S3YM000469 & $10: 43: 46.63$ & $-87: 25: 10.1$ & 9.68 & 3.586394 & LS & $\ldots$ & Binary? \\
\hline 003697 & S742000061 & $12: 08: 11.93$ & $-87: 35: 39.9$ & 11.55 & 26.065850 & LS & $\ldots$ & \\
\hline 003850 & S742000043 & $12: 34: 25.12$ & $-87: 34: 37.7$ & 10.14 & 14.928409 & P04 & $\ldots$ & [A] \\
\hline 004463 & S3YM000518 & $10: 40: 16.05$ & $-87: 29: 29.8$ & 11.16 & 0.434167 & $\mathrm{LS}$ & 55.5901 & Binary? \\
\hline 005954 & S742000078 & $12: 39: 58.23$ & $-87: 41: 37.2$ & 11.68 & 74.844696 & P04 & $\ldots$ & \\
\hline 008426 & S3YM000629 & $10: 14: 50.45$ & $-87: 36: 23.8$ & 11.10 & 8.768682 & $\mathrm{LS}$ & $\ldots$ & \\
\hline 009171 & S3YM000662 & $10: 12: 54.85$ & $-87: 38: 22.9$ & 13.95 & 0.591609 & LS & 55.9779 & $\mathrm{RRL},[\mathrm{A}]$ \\
\hline 009952 & S742000182 & $12: 43: 30.67$ & $-87: 53: 30.9$ & 11.39 & 23.675364 & P04 & $\ldots$ & [A] \\
\hline
\end{tabular}

Notes.

a Position: if the star is not in GSC2.3, the position is based on the CSTAR master image.

b Source of period: [LS], Lomb-Scargle method; [BLS], box fitting algorithm; [P04], Fourier decomposition with Period04 program.

${ }^{c}$ Epoch of primary eclipse or minimum light (when applicable), in JD-2454500.

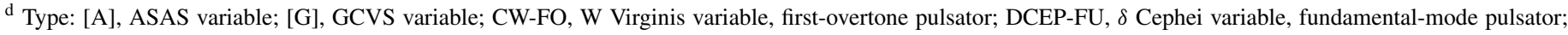

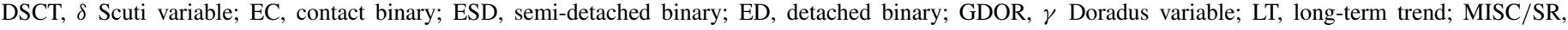
miscellaneous/semi-regular variable; RRL, RR Lyrae variable.

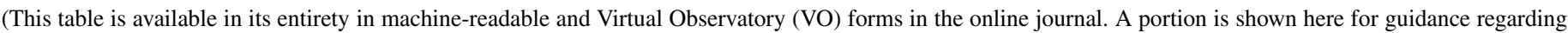
its form and content.)

We found 115 periodic variables by the LS method, 29 additional periodic variables via the Fourier decomposition technique, and 10 transit events through the BLS method, for a total of 154 periodic variables.

We phased the light curves of these stars using the most significant period and calculated the median value for every $10^{-3}$ in phase. We calculated the $L$ values for the binned phased light curves of these stars and compared the distribution to the one for 100 stars which had no significant periodicity. Each star in the latter sample was phased using all periods found in the former set, yielding $\sim 15,000$ test light curves. Figure 12 shows a comparison of both distributions. We selected 149 out of 154 objects with $L_{\mathrm{ph}}>0.25$ as our final sample of periodic variables. We further included eight variables with significant changes in magnitudes but no periodicity within our observing window (128 days). In these cases, we binned the data as a function of time every $3000 \mathrm{~s}$ and found values of $L_{\mathrm{ph}}>2$ in all cases.

The final sample of 157 variable stars is presented in Table 3 . Column 1 gives the CSTAR ID; Column 2 gives the ID from the Guide Star Catalog, version 2.3.2 (GSC2.3); Columns 3 and 4 give the right ascension and declination from GSC2.3; Column 5 gives the mean $i$-band magnitude; Column 6 gives the main period; and Column 7 gives a tentative classification of the variable type, when possible. Figure 13 shows the time series of three bright stars (one constant and two variables). Figure 14 shows folded light curves of a representative subset of the periodic variables, while Figures 15(a)-(f) show the time-series light curves for another representative subset of the periodic variables. Lastly, Figures 16(a)-(b) show two examples of variables with long-term trends but no periodicity in our observing window.

Twenty-eight CSTAR variables appear in previous variablestar catalogs: the ASAS (Pojmanski 2005) and the General Catalog of Variable Stars (GCVSs; Samus et al. 2009). The variables in common are listed in Table 4 . The periods we derived are in good agreement with previous determinations except for CSTAR\#032007, \#052891, and \#136863. The first

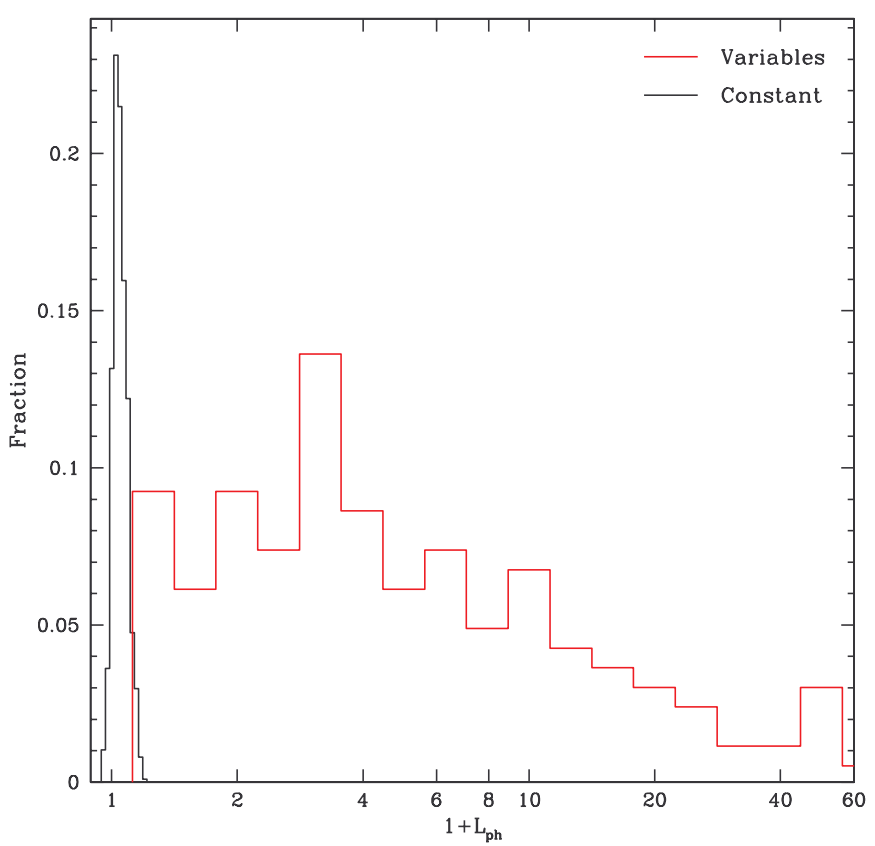

Figure 12. Histogram of variability statistic $L$ for objects that exhibited a significant periodicity using the LS technique (red), compared to the equivalent distribution for objects without a significant periodicity (black). The variability statistic was computed using phased light curves with $10^{3}$ points per cycle.

(A color version of this figure is available in the online journal.)

two variables have CSTAR periods that are $\sim$ twice the ASAS values, while the last one exhibits long-term variability in the CSTAR data which does not match the ASAS period.

We were unable to recover 14 previously known variables: 12 are saturated and 2 lie very close to saturated stars and were masked in our master frame. In the magnitude range $9.18<i<14.15$ that we have in common with GCVS and ASAS, we found five times more variable stars.

We classified approximately half of the variables into one of five types: binaries, $\delta$ Scuti, $\gamma$ Doradus, RR Lyrae, and objects 


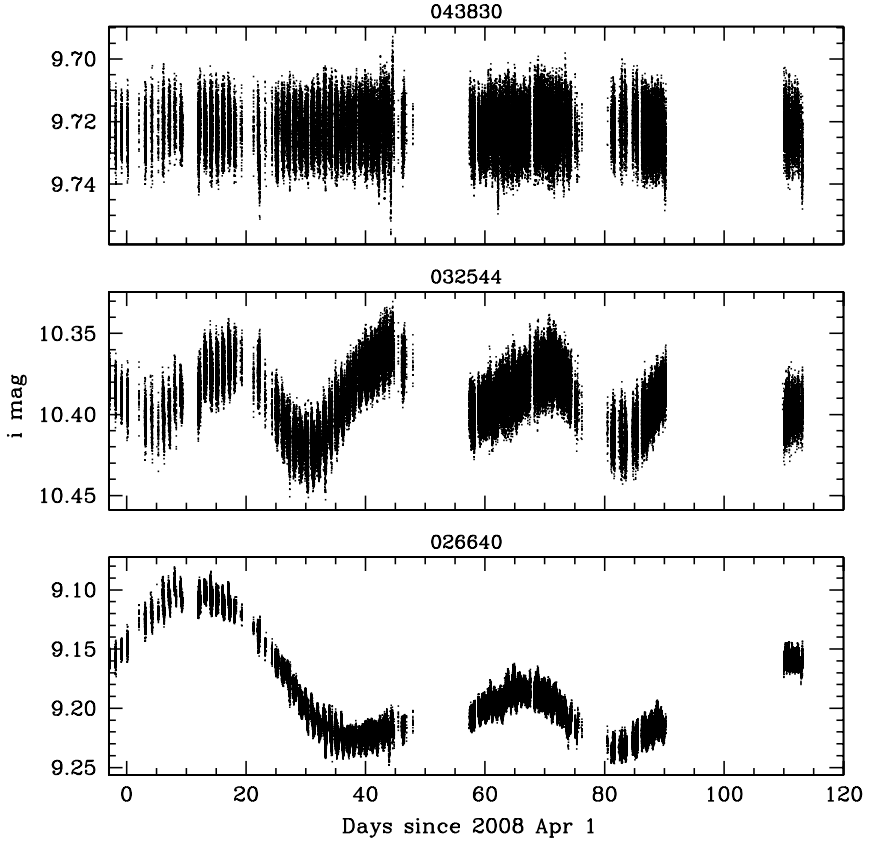

Figure 13. Light curves of a bright constant star (top panel) and two variable stars (bottom two panels).

with long-term variability which may be periodic beyond our observing window. One variable exhibits a light curve consistent with an exoplanet transit. Table 5 contains crude statistics for the different types.

\subsection{Search for Multiple Periods via Fourier Decomposition}

We searched for multiple periods in the time-series photometry of the variables using the Period04 program (Lenz \& Breger 2005). We started by identifying the frequency $\left(f_{1}\right)$ that displayed the highest $\mathrm{S} / \mathrm{N}$ peak in the periodogram. Next we prewhitened the time series (i.e., subtracted off the
Table 4

Variables in Common between CSTAR and Other Catalogs

\begin{tabular}{|c|c|c|c|}
\hline \multicolumn{2}{|r|}{ ID } & \multicolumn{2}{|c|}{$P$ (days) } \\
\hline CSTAR & Other & CSTAR & Other \\
\hline 001707 & [A] $123244-8726.4$ & 0.338528 & 0.338519 \\
\hline 003850 & [A]123423-8734.6 & 14.928409 & 18.63354 \\
\hline 009171 & [A] $101257-8738.4$ & 0.591609 & 0.59161 \\
\hline 009952 & [A]124330-8753.5 & 23.675364 & 23.71541 \\
\hline 013255 & [A]145422-8721.1 & LT & 174.6807 \\
\hline 020526 & [A]132341-8816.1 & 2.510798 & 2.51046 \\
\hline 022489 & [A]100123-8813.5 & 0.652255 & 0.65226 \\
\hline 026640 & [A]111701-8835.6 & 49.365707 & 51.8 \\
\hline 029379 & [A] $155703-8730.1$ & 3.102692 & 3.115622 \\
\hline 032007 & [A]092907-8829.7 & 0.621936 & 0.38355 \\
\hline 034724 & [A]100112-8844.6 & 42.966682 & 43.5 \\
\hline 034997 & [A]142904-8838.7 & 0.646586 & 0.64655 \\
\hline 036162 & [A]090354-8833.1 & 0.873470 & 0.87376 \\
\hline 038255 & [A]135318-8854.2 & 0.266903 & 0.266899 \\
\hline 038663 & [A]084613-8833.7 & 0.267127 & 0.267128 \\
\hline 052891 & [A]171319-8742.5 & 53.957802 & 29.036005 \\
\hline 055495 & [A]074400-8907.7 & 0.797670 & 0.79801 \\
\hline 057775 & [A]064047-8815.4 & 0.438611 & 0.43863 \\
\hline 071571 & [A] $054317-8804.1$ & LT & 426 \\
\hline 072730 & [A]182912-8832.6 & 0.573063 & 0.573034 \\
\hline 083768 & [A]051332-8719.7 & 0.384076 & 0.38408 \\
\hline 096404 & [A]194301-8746.9 & 0.581793 & 0.58171 \\
\hline 098092 & [A] $035443-8802.8$ & 89.237907 & 70.7 \\
\hline 110801 & [A]024230-8804.4 & 35.695164 & 47.6 \\
\hline 124666 & [A]214719-8739.1 & 0.458038 & 0.45803 \\
\hline 127850 & [G] ST Oct & LT & LT \\
\hline 133742 & [A] $223703-8728.8$ & 0.848378 & 0.84838 \\
\hline 136863 & [A] $230125-8722.3$ & LT & 37.31343 \\
\hline
\end{tabular}

Note. [A]: ASAS; [G]: GCVS; LT: long-term variability.

most significant frequency) and searched for the next highest peak in the frequency spectrum. We repeated the process until all peaks with $\mathrm{S} / \mathrm{N}>4$ were identified. For example, consider the variable CSTAR\#061353, shown in

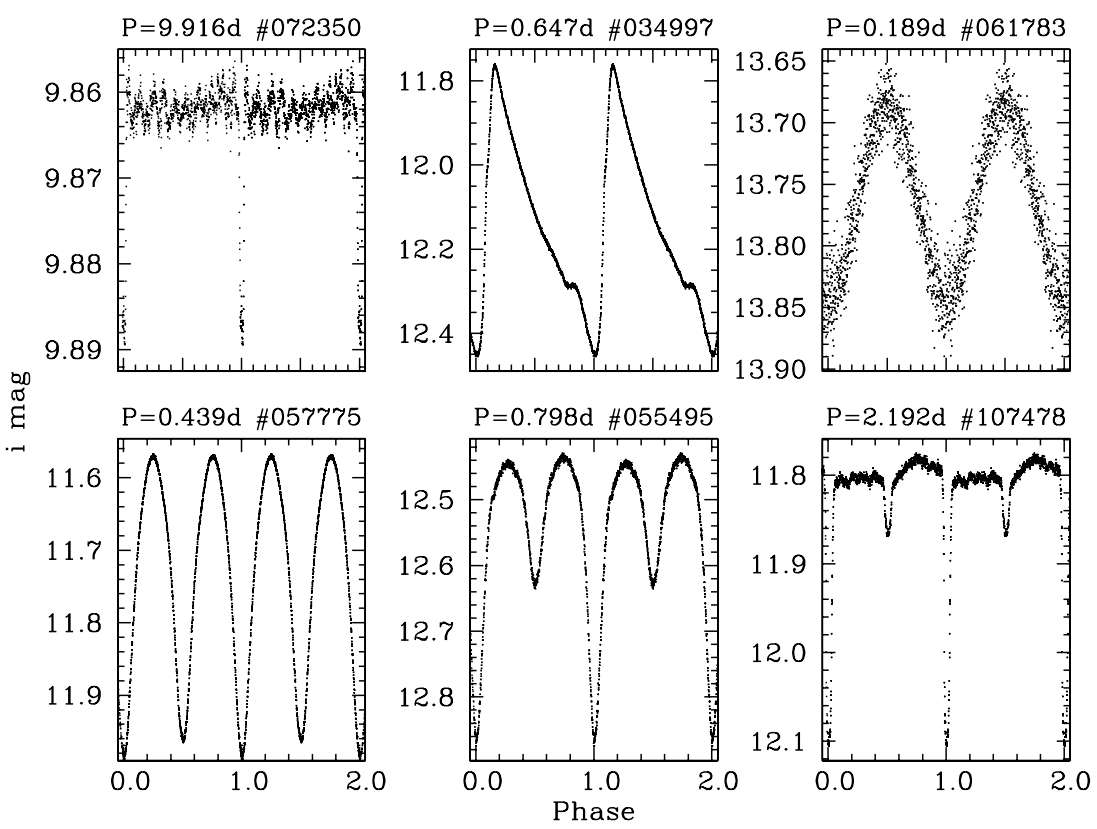

Figure 14. Phased light curves of six variable stars. The periods and CSTAR IDs are given in the figure. Top row, from left to right: transit variable; RR Lyrae; $\delta$ Scuti. Bottom row, from left to right: eclipsing binaries of contact (EC), semi-detached (ESD), and detached (ED) configurations. 

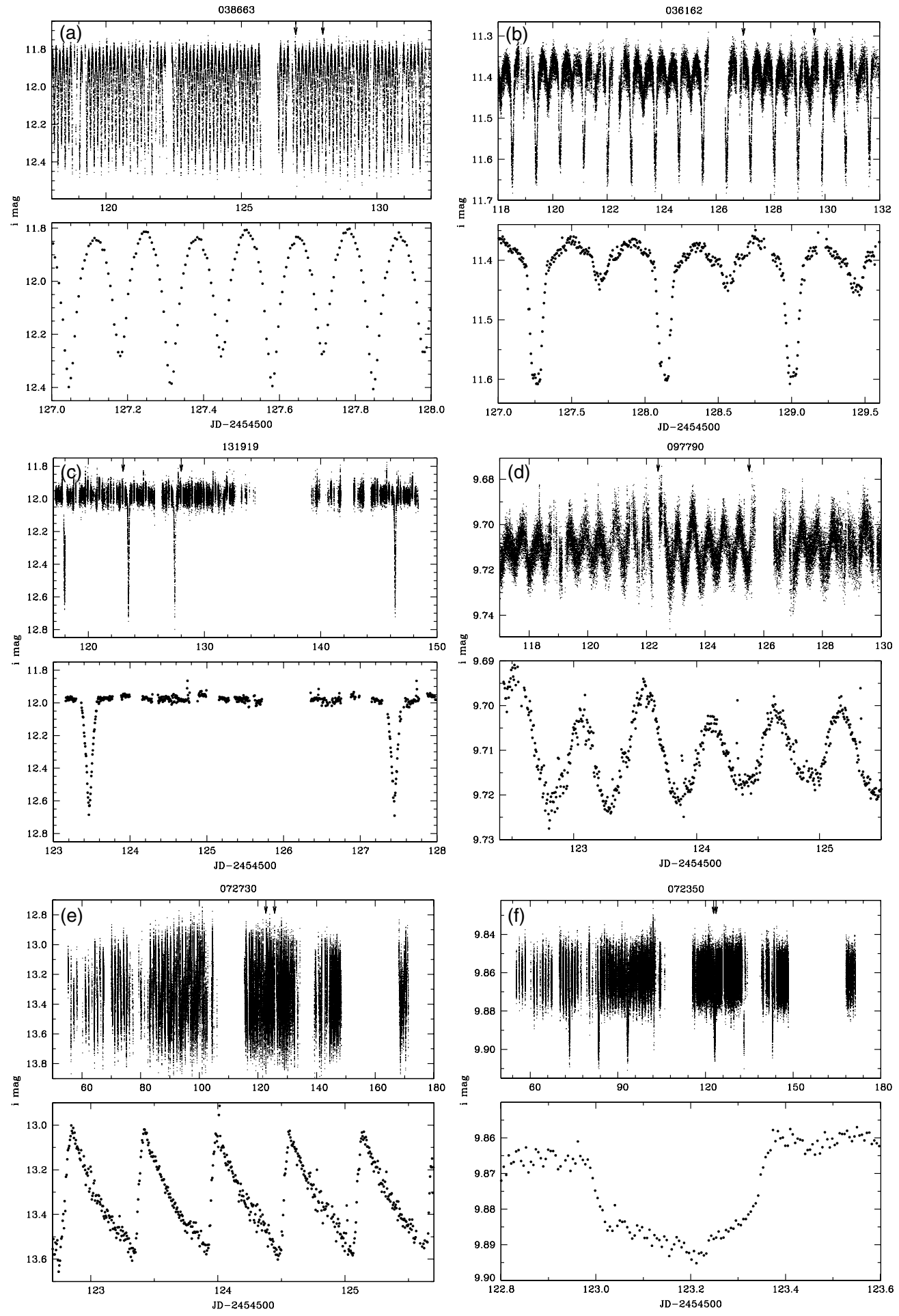

Figure 15. (a) Time-series light curve of a contact binary. Only a small fraction of the complete CSTAR data is shown. The top panel shows the light curve sampled at $20 \mathrm{~s}$ intervals. The bottom panel shows a portion of the top light curve (bounded by the arrows) binned into $450 \mathrm{~s}$ intervals. (b) Same as (a), but for a semi-detached binary. (c) Same as (a), but for a detached binary. (d) Same as (a), but for a variable of $\gamma$ Dor type. (e) Same as (a), but for a variable of RR Lyr type with Blazhko effect. (f) Same as (a), but for a possible transiting exoplanet.

Figure 17. There are three significant peaks in the periodogram, with $f_{i}=44.2879,44.1690$, and 42.1209 cycles day ${ }^{-1}$ ) and S/Ns of 15.8, 15.7, and 15.0, respectively. The top left inset shows the phased light curve for $f_{1}=44.2879$ cycles day $^{-1}$ with 1000 phase bins. Given the amplitude-frequency plot and the phased light curve, the dominant period of 32 minutes is likely real.
The Fourier decomposition results of all the periodic variables are listed in Table 6. We list in Columns 2-4 the frequency, amplitude (in mmag), and $\mathrm{S} / \mathrm{N}$ of each peak. Column 5 gives the relations between the primary and other frequencies. We do not list four transit-like variables with $P>6$ days $(072350$, $081845,097230,131919)$ for which we only have a period based on the BLS method. 

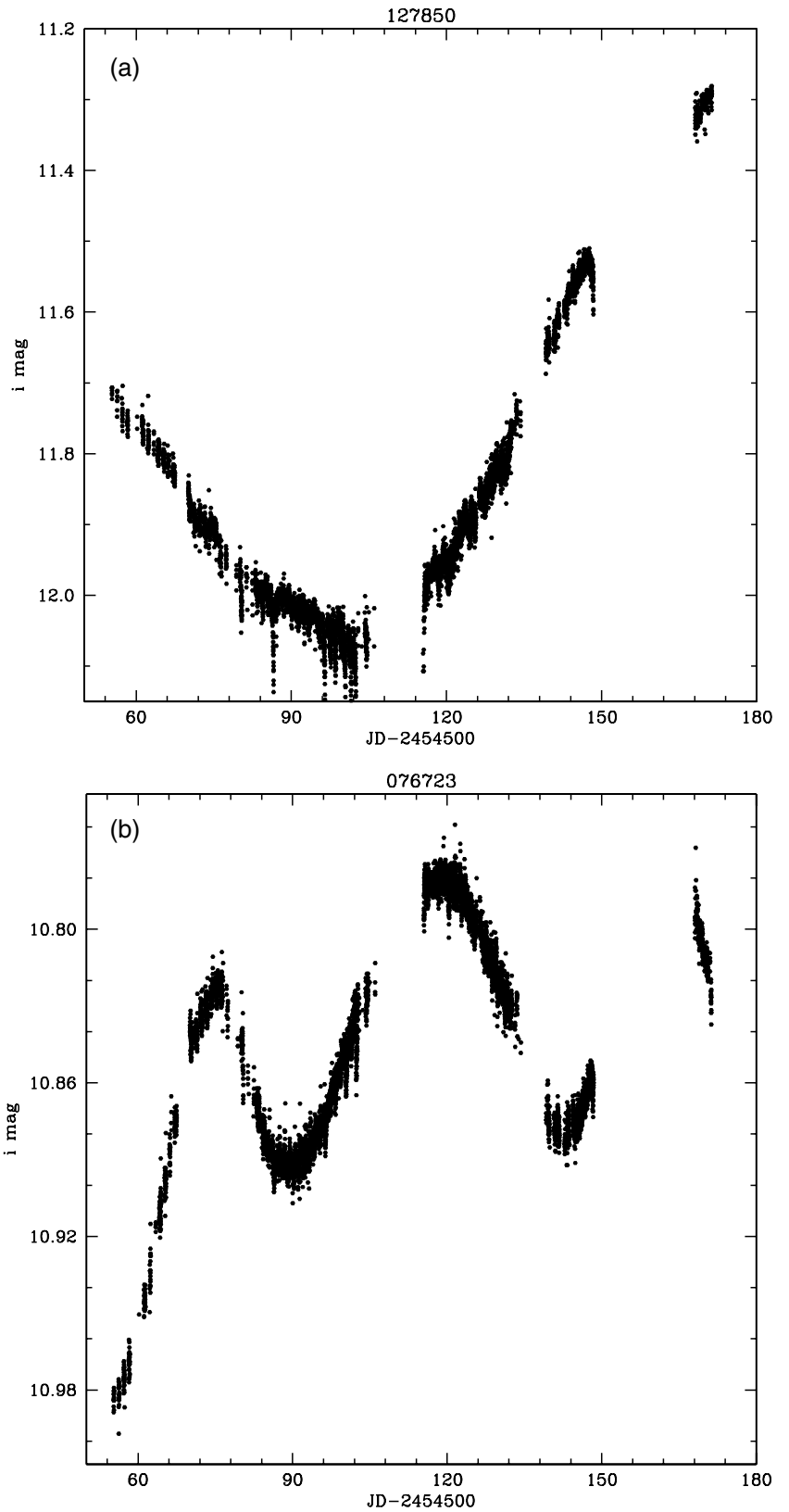

Figure 16. Complete CSTAR light curves for two long-term variables, binned at $450 \mathrm{~s}$ intervals.

\subsection{Types of Variables Found by CSTAR}

\subsubsection{Eclipsing Binaries}

Eclipsing binaries can be classified into three broad categories (Paczyński et al. 2006): contact (EC), semi-detached (ESD), and detached (ED). Typical light curves are shown in the three bottom panels of Figure 14 and in Figures 15(a)-(c).

There are 10 binaries among the 27 variables in common with ASAS (CSTAR\#001707, 020526, 022489036162,038255 , 038663, 055495, 057775, 083768, 133742). Their Fourier spectra show that the frequencies found are often integral or halfintegral multiples of each other. We identified an additional 31 variables exhibiting similar Fourier properties and light curves.

\subsection{2. $\delta$ Scuti Stars}

$\delta$ Scuti variables are late A- and early F-type stars situated in the instability strip on or above the main sequence in the H-R

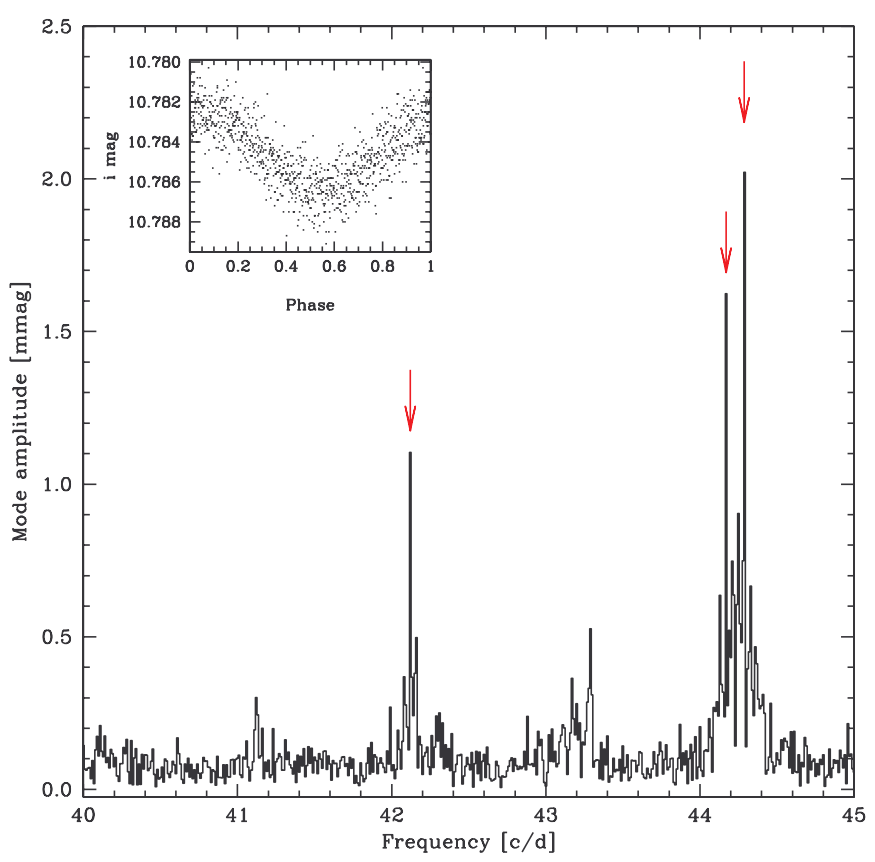

Figure 17. Fourier spectrum of variable candidate ID $=061353$ derived using the Period04 program. There are three significant peaks, at $f_{i}=44.288,44.169$, and 42.121 cycles day $^{-1}$. The inset on the top-left corner shows the light curve phased using $f_{i}=44.288$ cycles day $^{-1}$. See Table 6 for details.

(A color version of this figure is available in the online journal.)

Table 5

Distribution of Variable Star Types

\begin{tabular}{lrr}
\hline \hline Variable Type & $N$ & $\%$ \\
\hline Binary & 41 & 26.1 \\
$\delta$ Sct & 9 & 5.7 \\
Long-term & 8 & 5.1 \\
RR Lyr & 6 & 3.8 \\
$\gamma$ Dor & 3 & 1.9 \\
Transit & 1 & 0.6 \\
Unclassified & 89 & 56.7 \\
\hline
\end{tabular}

Diagram. Their typical pulsation periods are found to be in the interval of 0.02-0.25 days (Breger 2000). The frequencies of nine candidates are in the range 4-30 cycles day ${ }^{-1}$ such as star 061783 in Table 6.

\subsection{3. $\gamma$ Doradus Stars}

$\gamma$ Doradus variables constitute a class of multi-periodic pulsating variables that have a strong asteroseismic potential since they are located in a region of the H-R Diagram where $g$-mode pulsations, $p$-mode pulsations, and solar-like variability may exist in a single star (Kaye et al. 1999). They are of spectral type $\mathrm{A}$ or $\mathrm{F}$ and are located on or just above the main sequence. Observationally, they exhibit multiple periods between 0.3 and 3 days, with typical photometric amplitudes of 5-50 mmag (Cuypers et al. 2009). Based on the Fourier decomposition, three candidates are likely $\gamma$ Dor stars. Star 070941 has three frequencies of $1.65,1.60$, and 1.83 cycles day $^{-1}$. Its spectral type is F0 from the Tycho-2 Spectral Type Catalog (Wright et al. 2003). It is located at R.A. $=5^{\mathrm{h}} 47^{\mathrm{m}} 8^{\mathrm{s}} .05$, decl. $-87^{\circ} 51^{\prime} 0^{\prime \prime} .17$ (J2000). To our knowledge there are no published spectra of the other two candidates (081723 and 097790 shown in Figure 15(d)). 
Table 6

Fourier Analysis of Variable Star Candidates

\begin{tabular}{|c|c|c|c|c|}
\hline $\begin{array}{l}\text { CSTAR } \\
\text { ID }\end{array}$ & $\begin{array}{c}\text { Frequency } \\
\left(\text { cycles day }^{-1}\right)\end{array}$ & $\begin{array}{l}\text { Amplitude } \\
\text { (mmag) }\end{array}$ & $\mathrm{S} / \mathrm{N}$ & Notes \\
\hline 000572 & $\begin{array}{r}6.475955 \\
16.964241 \\
\end{array}$ & $\begin{array}{l}60.74 \\
11.56 \\
\end{array}$ & $\begin{array}{r}11.04 \\
4.26 \\
\end{array}$ & \\
\hline 001707 & $\begin{array}{r}5.907794 \\
11.815589 \\
17.723383 \\
23.631178 \\
2.953241 \\
29.539444\end{array}$ & $\begin{array}{r}259.90 \\
94.63 \\
43.02 \\
16.74 \\
13.88 \\
7.67\end{array}$ & $\begin{array}{r}19.84 \\
19.71 \\
20.49 \\
18.22 \\
7.30 \\
15.23\end{array}$ & $\begin{array}{c}\mathrm{f} 1 \\
2 \mathrm{f} 1 \\
3 \mathrm{f} 1 \\
4 \mathrm{f} 1 \\
0.5 \mathrm{f} 1 \\
5 \mathrm{f} 1\end{array}$ \\
\hline 003125 & $\begin{array}{l}0.278487 \\
0.139674 \\
5.016641\end{array}$ & $\begin{array}{r}22.41 \\
16.74 \\
5.56\end{array}$ & $\begin{array}{l}8.53 \\
8.38 \\
4.27\end{array}$ & $\begin{array}{c}\mathrm{f} 1 \\
0.5 \mathrm{f} 1\end{array}$ \\
\hline 003697 & 0.037071 & 33.69 & 13.28 & \\
\hline 004463 & $\begin{array}{r}2.303088 \\
5.013947 \\
4.604452 \\
6.905816 \\
9.206318 \\
11.510546 \\
13.812341 \\
13.032144 \\
16.113611 \\
26.071184 \\
34.092800\end{array}$ & $\begin{array}{l}7.66 \\
9.09 \\
6.45 \\
6.21 \\
4.97 \\
3.98 \\
2.76 \\
2.50 \\
2.18 \\
1.27 \\
1.02\end{array}$ & $\begin{array}{l}4.74 \\
5.98 \\
5.49 \\
6.45 \\
6.43 \\
7.64 \\
6.52 \\
6.10 \\
5.81 \\
4.27 \\
4.33\end{array}$ & $\begin{array}{l}\mathrm{f} 1 \\
2 \mathrm{f} 1 \\
3 \mathrm{f} 1 \\
4 \mathrm{f} 1 \\
5 \mathrm{f} 1 \\
6 \mathrm{f} 1\end{array}$ \\
\hline 005954 & $\begin{array}{l}0.013361 \\
0.051720\end{array}$ & $\begin{array}{l}37.42 \\
10.34\end{array}$ & $\begin{array}{r}16.30 \\
7.87\end{array}$ & \\
\hline 008426 & 0.113784 & 10.93 & 7.71 & \\
\hline 009171 & $\begin{array}{r}1.690393 \\
3.380355 \\
5.070748 \\
6.761141 \\
8.451533 \\
10.141927\end{array}$ & $\begin{array}{r}270.20 \\
139.87 \\
90.51 \\
67.58 \\
42.15 \\
20.77\end{array}$ & $\begin{array}{r}10.48 \\
10.87 \\
10.35 \\
10.71 \\
9.40 \\
5.92\end{array}$ & $\begin{array}{l}\mathrm{f} 1 \\
2 \mathrm{f} 1 \\
3 \mathrm{f} 1 \\
4 \mathrm{f} 1 \\
5 \mathrm{f} 1 \\
6 \mathrm{f} 1\end{array}$ \\
\hline 009952 & 0.042238 & 30.29 & 11.46 & \\
\hline 011616 & 0.031463 & 17.31 & 10.59 & \\
\hline
\end{tabular}

(This table is available in its entirety in machine-readable and Virtual Observatory (VO) forms in the online journal. A portion is shown here for guidance regarding its form and content.)

\subsubsection{RR Lyrae Stars}

RR Lyrae are low-mass stars undergoing helium core fusion with spectral types ranging from A2 to F6 (Smith 1995). They exhibit periods from $\sim 0.2$ to $\sim 1$ day and photometric amplitudes in the optical bands of 0.3-2 mag. Most RR Lyrae stars pulsate in the radial fundamental mode (RRab stars). RRc stars pulsate radially in the first overtone. RRd stars, which are much rarer, pulsate in both modes simultaneously. There are six RRAB stars in our sample (009171, 032007, 034997, 072730, 096404, 124666), all of which were previously identified by ASAS. Additionally, CSTAR\#032007 is listed in the GCVS as W Oct.

Some RR Lyrae stars show modulations in their period, pulsation amplitude, light curve shape, and radial velocity curves. These modulations are known as the "Blazhko effect" (Blažko 1907; Buchler \& Kolláth 2011). Previous surveys have found different percentages of RR Lyrae exhibiting the Blazhko effect: $10 \%-30 \%$ of RRab variables in Alcock et al. (2003) and 50\% in Jurcsik et al. (2009). The Fourier spectrum of a Blazhko-effect RR Lyrae is characterized by triplets, quintuplets, and high-order multiplets around the pulsation frequency components.

Based on the period analysis in Table 6 and light curve shapes we found that 4 out of 6 RR Lyr stars in our sample exhibit the Blazhko effect (034997, 072730, 124666, 032007). One such star is shown in Figure 15(e).

\subsubsection{Other Types of Variables}

Variable CSTAR\#072350 displays a transit-like light curve with a period of 9.916 days and a depth of 17 mmag, shown in the top left panel of Figure 14 and Figure 15(f). The primary is a bright F5 star with $T_{\text {eff }} \sim 6400 \mathrm{~K}$ from the Tycho-2 Spectral Type Catalog (Wright et al. 2003). If confirmed, this would be an interesting addition to the known extrasolar planets given the relatively long orbital period and temperature of the primary.

There are eight variable candidates with long-term trends, which may have periods longer than the observing window of 128 days. Some are probably Mira stars, such as CSTAR\#127850 which shows an amplitude variation of 1.4 mag. Its light curve is plotted in Figure 16(a).

89 variable candidates have no classification in Column 6 of Table 3; half of these were previously unknown. Follow-up photometric and spectroscopic observations will be required to determine their nature. One of these variables, CSTAR\#065072, has been spectroscopically identified as an M3.5 dwarf in the solar neighborhood by Riaz et al. (2006).

\subsection{Blending}

The large pixel scale of the camera and the relatively high stellar density of the field make blending a problem. We define a blend as two or more stars located within $30 \operatorname{arcsec}(\sim 2$ CSTAR pixels) of each other. Inspection of DSS images reveals that $\sim 1 / 3$ of the candidate variable stars are blended with one neighboring star while $\sim 6 \%$ are blended with two or more neighboring stars. Follow-up photometry with a finer pixel scale will be required to ensure that these blends are not responsible for the observed variability. Future telescopes planned for Dome A will yield a considerably finer pixel scale which will mitigate this problem (AST3; Cui et al. 2008). Additionally, we are implementing a difference-imaging photometry pipeline that will enable a more robust detection of variables in crowded environments and will deliver higher photometric precision.

\section{SUMMARY}

CSTAR has a large field of view $\left(23 \mathrm{deg}^{2}\right)$ and the ability to provide uninterrupted observations for the entire duration of the Antarctic winter night. We have obtained high-quality time-series photometry for 10,000 stars with $i<14.5$ mag and detected 157 variables, $5 \times$ more than previous surveys of same area of the sky in the same magnitude range.

Our photometry indicates that Dome $\mathrm{A}$ is a viable and excellent observing site. During the 2008 Antarctic winter season, $96 \%$ of the images obtained at a solar elevation angle below $-10^{\circ}$ were useful for scientific purposes. The median sky background in the $i$ band was 19.6 mag per $\operatorname{arcsec}^{2}$ and the median extinction due to clouds was below $0.1 \mathrm{mag}$.

Lingzhi Wang acknowledges financial support by the China Scholarship Council and the National Natural Science Foundation of China under the Distinguished Young Scholar Grant 
10825313 and Grant 11073005 , by the Ministry of Science and Technology National Basic Science Program (Project 973) under grant number 2007CB815401, and by the Excellent Doctoral Dissertation of Beijing Normal University Engagement Fund.

Lucas Macri and Lifan Wang acknowledge support by the Department of Physics \& Astronomy at Texas A\&M University through faculty startup funds and the Mitchell-Munnerlyn-Heep Chair for tenure-track faculty.

This work was supported by the Chinese PANDA International Polar Year project, NSFC-CAS joint key program through grant number 10778706 , CAS main direction program through grant number KJCX2-YW-T08. The authors deeply appreciate the great efforts made by the 24-27th Dome A expedition teams who provided invaluable assistance to the astronomers that set up and maintained the CSTAR telescope and the PLATO system. PLATO was supported by the Australian Research Council and the Australian Antarctic Division. Iridium communications were provided by the US National Science Foundation and the US Antarctic Program.

\section{REFERENCES}

Alcock, C., Alves, D. R., Becker, A., et al. 2003, ApJ, 598, 597

Ashley, M. C. B., Allen, G., Bonner, C. S., et al. 2010, Highlights Astron., 15, 627

Baglin, A., Auvergne, M., Boisnard, L., et al. 2006, in 36th COSPAR Scientific Assembly, Vol. 36, 3749

Benkő, J. M., Kolenberg, K., Szabó, R., et al. 2010, MNRAS, 409, 1585

Blažko, S. 1907, Astron. Nachr., 175, 325

Boisnard, L., \& Auvergne, M. 2006, in Proc. The CoRoT Mission Pre-Launch Status-Stellar Seismology and Planet Finding, ed. M. Fridlund, A. Baglin, J. Lochard, \& L. Conroy (ESA Special Publication, Vol. 1306), 19

Bonner, C. S., Ashley, M. C. B., Cui, X., et al. 2010, PASP, 122, 1122

Borucki, W. J., Koch, D., Basri, G., et al. 2010, Science, 327, 977

Breger, M. 2000, in ASP Conf. Ser. 210, Delta Scuti and Related Stars, ed. M. Breger \& M. Montgomery (San Francisco, CA: ASP), 3

Buchler, J. R., \& Kolláth, Z. 2011, ApJ, 731, 24

Burton, M. G. 2010, A\&AR, 18, 417

Crouzet, N., Guillot, T., Agabi, A., et al. 2010, A\&A, 511, A36

Cui, X., Yuan, X., \& Gong, X. 2008, Proc. SPIE, 7012, 70122D

Cuypers, J., Aerts, C., De Cat, P., et al. 2009, A\&A, 499, 967

Fukugita, M., Ichikawa, T., Gunn, J. E., et al. 1996, AJ, 111, 1748

Hengst, S., Allen, G. R., Ashley, M. C. B., et al. 2008, Proc. SPIE, 7012, 70124E
Jurcsik, J., Sódor, Á., Szeidl, B., et al. 2009, MNRAS, 400, 1006

Kaluzny, J., Stanek, K. Z., Krockenberger, M., et al. 1998, AJ, 115, 1016

Kaye, A. B., Handler, G., Krisciunas, K., Poretti, E., \& Zerbi, F. M. 1999, PASP, 111,840

Kenyon, S. L., Lawrence, J. S., Ashley, M. C. B., et al. 2006, PASP, 118, 924

Kenyon, S. L., \& Storey, J. W. V. 2006, PASP, 118, 489

Kovács, G., Zucker, S., \& Mazeh, T. 2002, A\&A, 391, 369

Lasker, B. M., Lattanzi, M. G., McLean, B. J., et al. 2008, AJ, 136, 735

Lawrence, J. S., Allen, G. R., Ashley, M. C. B., et al. 2008, Proc. SPIE, 7012, 701227

Lawrence, J. S., Ashley, M. C. B., Burton, M. G., et al. 2006, Proc. SPIE, 6267, $62671 \mathrm{~L}$

Lawrence, J. S., Ashley, M. C. B., Hengst, S., et al. 2009, Rev. Sci. Instrum., 80,064501

Lenz, P., \& Breger, M. 2005, Commun. Asteroseismol., 146, 53

Lomb, N. R. 1976, Ap\&SS, 39, 447

Luong-van, D. M., Ashley, M. C. B., Cui, X., et al. 2010, Proc. SPIE, 7733, $77331 \mathrm{~T}$

Monet, D. G., Levine, S. E., Canzian, B., et al. 2003, AJ, 125, 984

Mosser, B., \& Aristidi, E. 2007, PASP, 119, 127

Ofek, E. O. 2008, PASP, 120, 1128

Paczyński, B., Szczygieł, D. M., Pilecki, B., \& Pojmański, G. 2006, MNRAS, 368, 1311

Pojmanski, G. 2005, VizieR On-line Data Catalog (originally published in Pojmanski, G. 2000, Acta Astron., 50, 177)

Riaz, B., Gizis, J. E., \& Harvin, J. 2006, AJ, 132, 866

Samus, N. N., Durlevich, O. V., Kazarovets, E. V., et al. 2009, General Catalogue of Variable Stars (Moscow: Moscow State Univ.)

Saunders, W., Lawrence, J. S., Storey, J. W. V., et al. 2009, PASP, 121, 976

Saunders, W., Lawrence, J. S., Storey, J. W. V., et al. 2010, in EAS Pub. Ser. 40 , Proc. 3rd ARENA Conf.: An Astronomical Observatory at CONCORDIA (Dome C, Antarctica), ed. L. Spinoglio \& N. Epchtein, 89

Scargle, J. D. 1982, ApJ, 263, 835

Smith, H. 1995, RR Lyrae Stars (Cambridge: Cambridge Univ. Press)

Stetson, P. B. 1987, PASP, 99, 191

Stetson, P. B. 1996, PASP, 108, 851

Storey, J. W. 2009, Assoc. Asia-Pac. Phys. Soc. Bull., 19, 13

Storey, J. W. V. 2005, Antarct. Sci., 17, 555

Storey, J. W. V. 2007, Chin. Astron. Astrophys., 31, 98

Strassmeier, K. G., Briguglio, R., Granzer, T., et al. 2008, A\&A, 490, 287

Swain, M. R., \& Gallée, H. 2006, PASP, 118, 1190

Taylor, M. 1990, AJ, 100, 1264

Wright, C. O., Egan, M. P., Kraemer, K. E., \& Price, S. D. 2003, AJ, 125, 359

Yang, H., Allen, G., Ashley, M. C. B., et al. 2009, PASP, 121, 174

Yuan, X., Cui, X., Liu, G., et al. 2008, Proc. SPIE, 7012, 70124G

Zhou, X., Fan, Z., Jiang, Z., et al. 2010a, PASP, 122, 347

Zhou, X., Wu, Z., Jiang, Z., et al. 2010b, Res. Astron. Astrophys., 10, 279

Zou, H., Zhou, X., Jiang, Z., et al. 2010, AJ, 140, 602 